\title{
How to approach patients in the orthodontic surgical protocol? Patient access in the orthodontic surgical protocol
}

\author{
1 Specialist certified in dentofacial orthopedics \\ 2 Maxillofacial surgeon \\ 3 Hospital practitioner \\ ${ }_{4}$ Private practice
}

M. Boukili Makhoukhi ${ }^{1,3}$, M. Makaremi ${ }^{1,4}$, M. El Okeily

\begin{abstract}
The ortho-surgical patient is one who requires special care, this is often a source of apprehension for the orthodontist. Indeed, beyond implementing the right solution and controlling the technical aspect of the treatment, it puts the practitioner in a position of coordinator of a multidisciplinary team, focused on a patient requesting a lot of information and attention. Patient approach is a long process, it can be divided into two phases. The first phase is one of initial information and patient orientation intended for the different actors of the protocol, namely a maxillofacial surgeon, a physiotherapist or a speech therapist. A psychological support is also necessary, either from the patient's family and/or from a psychotherapist. Then, after careful consideration and informed consent from the patient, the second practical phase of the ortho-surgical protocol begins, once more, with a high level of patient/ practitioner interaction, in order to prevent the patient from falling into the traps of e-medicine.
\end{abstract}

\section{KEYWORDS}

Multidisciplinary treatment, orthognathic surgery, psychological approach, e-medicine

\section{INTRODUCTION}

The impression made during the initial consultation with a patient and the subsequent practitioner-patient relationship that develops are key to the success of orthodontic surgical treatment. Unfortunately, too often, orthodontists and surgeons focus on the technical aspect of the protocol, thereby neglecting how patients may be affected by the process, particularly after being exposed to the onslaught of reviews available on the Internet.

Before engaging in the orthodontic surgical protocol, patients ask for very precise information, often in response to what they

Address for correspondence:

M. Boukili Makhoukhi

40 rue de Mesly - 94000 Créteil

E-mail: mariambouk@gmail.com
Article received: 07-01-2016. Accepted for publication: 20-02-2016.

This is an Open Access article distributed under the terms of the Creative Commons Attribution License (http://creativecommons.org/licenses/by/4.0), which permits unrestricted use, distribution, and reproduction in any medium, provided the original work is properly cited. 
read on various blogs and discussion forums. From the very first consultation, it is our role and responsibility to address their concerns and to try to alleviate their fears (particularly, surgical). We must also ensure holistic, multidisciplinary patient care, which will involve a physiotherapist, speech therapist, the patient's relatives, and/ or a psychotherapist in addition to an orthodontist and a surgeon.

In this article, we will detail what ideally happens during initial contact with patients, which includes making a diagnosis, identifying at-risk patients,

\section{THE INITIAL CONTACT WITH THE PATIENT}

The manner in which the patient is first approached during the orthodontic surgical protocol is the most important phase of the procedure. Sufficient time must be allotted to analyze the patient's requests and then to introduce the other care providers involved in the protocol. The aim of all this is to allow the patient sufficient time to reflect before definitively committing to the proposed treatment.

The patient's requests are therefore crucial. The orthodontist will not approach an adult patient with a specific request such as an overly protruding jaw in the same way that he/she would address a young adolescent accompanied by his/her parents who complains of protruding teeth. Most cases are like the first case. The first case specified here concerns a patient who already had orthodontic treatment and who was concerned about esthetics and function and probably already heard of the need for surgery. The second case concerns a patient providing useful information, and introducing patients to the other participants in the surgery. We will then look at the practitioner's approach during the orthodontic surgical protocol insisting on the importance of providing sufficient information and psychological support. We will substantiate this article with a satisfaction survey conducted after performing bimaxillary surgery, which was distributed to 300 patients who had their operations at Tondu clinic in Bordeaux on January $15^{\text {th }}$ and based on an analysis of 75 questionnaires deemed admissible.

whose goal was to have the teeth aligned with braces.

Vanessa, aged 37 (fig. 1), already had orthodontic treatment during adolescence where four premolars were extracted. While being addressed by her periodontist during the maintenance phase of severe gum disease, she was aware of the need to undergo the surgery that her parents refused to give consent to some years before. She was a bit bothered by her gummy smile but she detested her double chin that she should not have had at her age. After the second orthodontic assessment, she was referred to a maxillofacial surgeon to validate the indication of mandibular advancement surgery. Regularly followed up by her periodontist, she was very enthusiastic, assiduous, and motivated throughout orthodontic preparation and did not show any sign that she needed psychological support.

Postoperatively, this enthusiasm waned as she slowly adapted to her new 

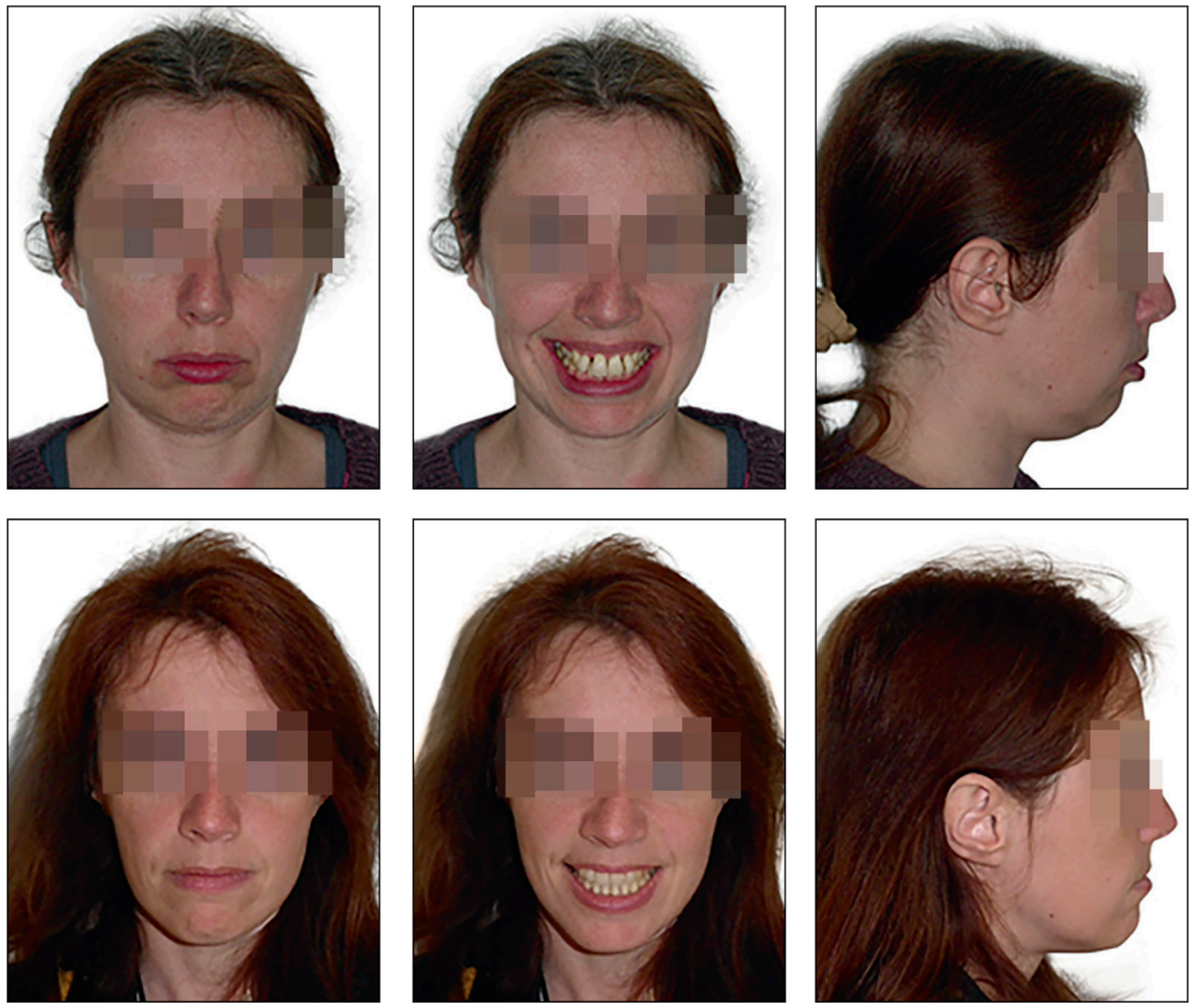

Figure 1

Vanessa, aged 37.

face. She would have had a better experience of the orthodontic surgical protocol had she been referred to a psychotherapist before beginning treatment.

Jordan, aged 19, (fig. 2), came to an orthodontic consultation complaining of severe mandibular overcrowding. Orthodontic surgical treatment with the extraction of four premolars was proposed and confirmed by the surgeon. Orthodontic preparation followed by mandibular advancement took place without any mishaps. Fifteen days after the surgery, nothing changed; he is very satisfied. His mouth opening is normal, and his orthodontic finishes are rapidly underway.
Orthodontists should therefore listen to and evaluate patients' requests and determine the best way to address surgical intervention while precluding any excessive and unrealistic expectations or a passive agreement from the patient who will simply "endure" and not actively engage in the orthodontic surgical protocol. Patients should therefore be given the necessary time to begin the protocol. In our satisfaction survey, $37.68 \%$ of patients admitted to having been informed of the surgery more than a year before the start of orthodontics and $46.38 \%$ had heard it at the beginning of orthodontic treatment (fig. 3). 

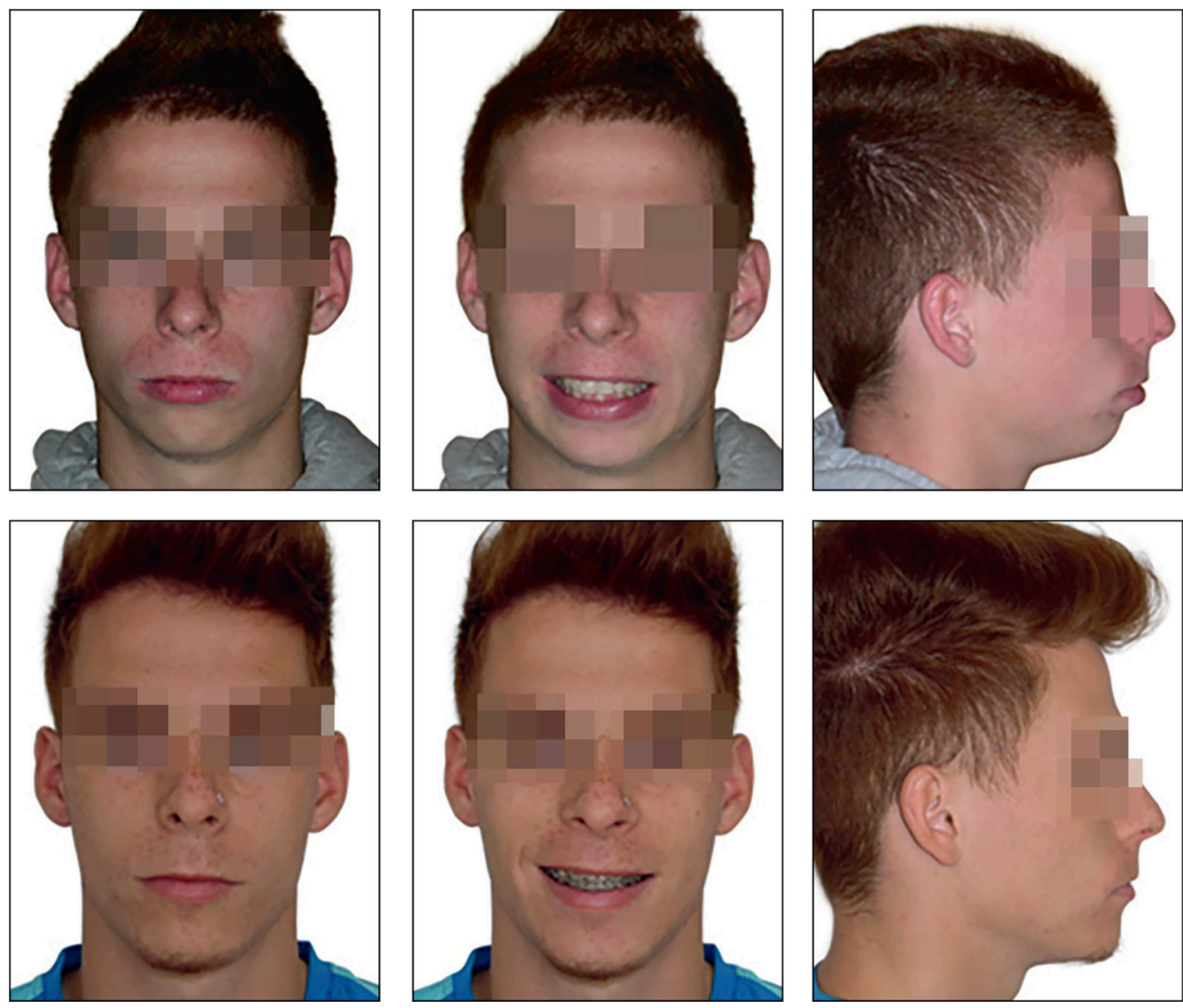

Figure 2

Jordan, age 19.

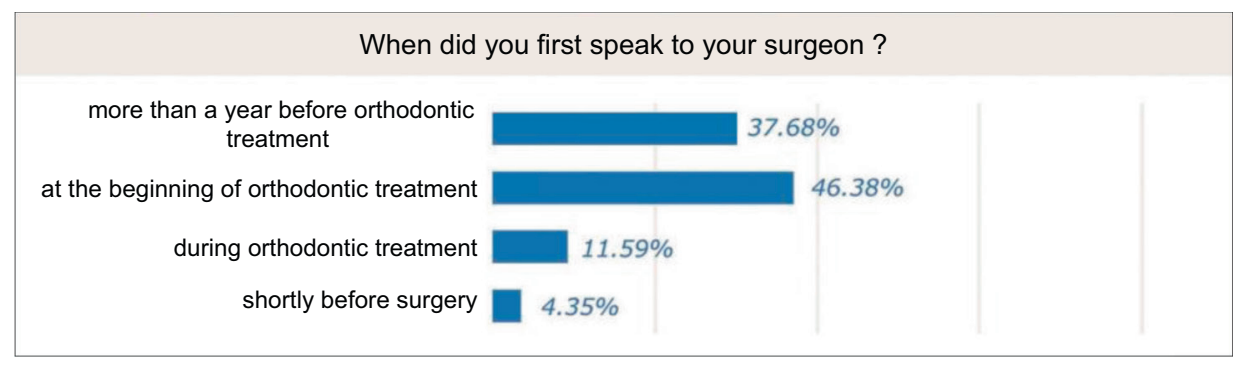

Figure 3

Statistical analysis of the first question of the satisfaction questionnaire. 
Among the more common motives for patients undergoing orthodontic surgical treatment, studies report functional motives with an improvement in dental occlusion and mastication for $49 \%$ of the patients. In total, $48 \%$ of those with esthetic motives have witnessed noticeable improvements in their teeth and $40 \%$ have reported an improvement in their facial profile $1,9,16$. However, the survey has revealed an improvement in their personal sense of well-being as indicated by $49 \%$ of the patients. This notion of well-being must be seriously considered; if not, a practitioner could fail to diagnose a patient's body dysmorphic disorder $(\mathrm{BDD})^{3,7,10,12}$

BDD is defined by pervasive fixation on a well-defined body part deemed defective in appearance. Patients therefore await medical confirmation as the perceived defects are not visible to a non-expert eye. This psychological disorder will cause dissatisfaction at the end of the orthodontic surgical protocol. It is therefore important to identify patients who may be at risk beforehand by asking a very specific set of questions that are aimed toward the detection of BDD ${ }^{11}$. Some of them are follows: In your opinion, how severe are your body flaws? How much time do you spend per day thinking about your flaw? What is the daily impact of this flaw? Are you socially or professionally handicapped by it?

Have you already had undergone or asked about esthetic surgery? Were you satisfied with it?

Should there be any doubt vis-a-vis the presence of this personality type, the patient should be referred to a psychotherapist who will provide a specialist perspective regarding the presumed

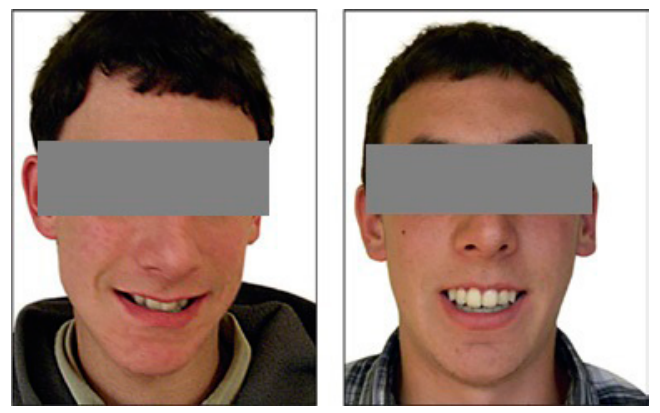

Figure 4

Fabien 18 ans.

benefits by carefully analyzing the initial request ${ }^{14,4}$. The patient will then be able to benefit from a forum in which he/ she can freely express his/her personal difficulties with the medical discourse, and this can progressively happen in a medium-to-long-term psychological follow-up.

Fabien attended his first consultation at age 18 (fig. 4); his mother reported that his social isolation was attributed to many complex esthetic factors, particularly persistent acne and facial asymmetry. The first photograph of Fabien's face is difficult to obtain because his gaze is averted and his smile forced. At the end of treatment and owing to psychological support, Fabien now has more self-confidence and his social life has certainly improved. The same is true for Clara (fig. 5), whose asymmetry is at the root of complex esthetic features that she had to live with since adolescence. She had undergone delayed treatment until she was aged 24. Orthodontic surgical treatment softened her face and reinvigorated her gaze, but her psychological follow-up helped her embrace her new face and caused her to blossom socially and professionally.

After analyzing the initial request, a practitioner should ensure the 

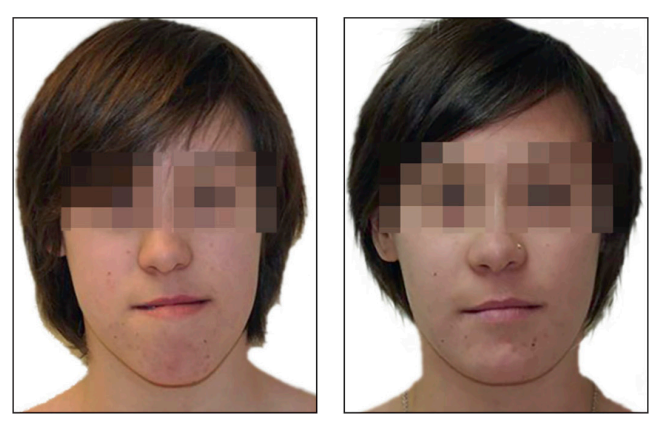

Figure 5

Clara, aged 24.

reliability of the patient's source of information. When an orthodontist announces the diagnosis, patient should be presented with an information pamphlet that details orthognathic surgery. We recommend the documentation made available from the University Hospital of Tours (http://www.chutours.fr/la-chirurgie-orthognatiquedocumentation.html) as well as the information booklet for patients from Global D: orthognathic surgery. The information is summative and general but remains invaluable to those who have to undergo surgery for the first time. As a matter of fact, $92.96 \%$ (fig. 6) of our patients have judged the information to be satisfactory and $58.67 \%$ believed that they received the information at the right time (fig. 7). Surgical information will be specified and individualized by a surgeon who possesses simulation software and, more importantly,

\section{The information provided by your orthodontist seemed}

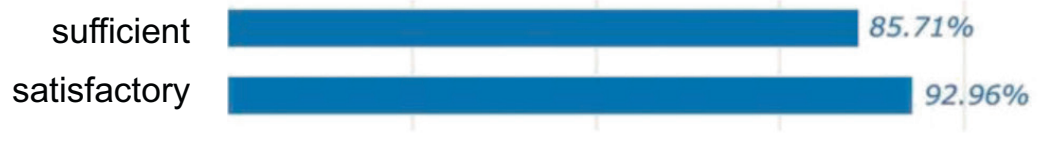

Figure 6

Statistical analysis of the second question on the satisfaction questionnaire.

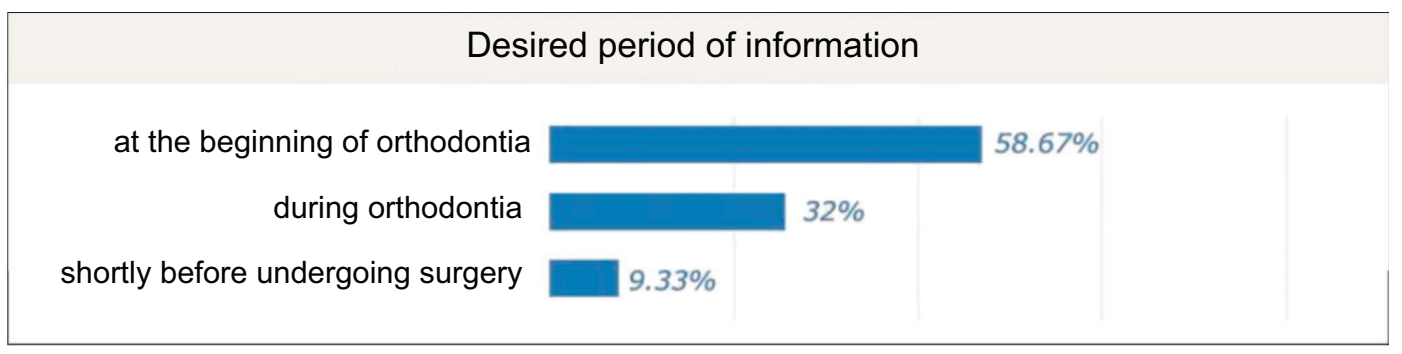

Figure 7

Statistical analysis of the third question on the satisfaction questionnaire. 
photographs of patients having the same dysmorphosis and who have already received treatment.

The ideal situation would be having two consultations at more than 3-week intervals to facilitate a sufficient time lapse during time which a patient can definitively confirm their consent to undergo surgery. The statistical analysis of our 75 questionnaires showed that $63.51 \%$ of patients acquiesce to undergo surgery but remain apprehensive (fig. 8).

Finally, patients should be sensitized regarding the literature available on the Internet and should avoid falling victim to the shortcomings or deficiencies of cybermedicine. While it is true that professional health websites contain reliable information that may be adapted to an individual context, personal blogs are much less reliable or appropriate.

However, these "reviews" continue to multiply, perhaps because they respond to patients' needs to express what they feel and to publicly share their pain and thereby feel less alone during this process. Therefore, on his website, https://machirurgieorthognatique.wordpress.com,
Alex keeps a detailed journal that is supported by photos, chronicling his process from his admission to the hospital until the day the plates were removed: " 2.5 years post-bimax + genio and 1.5 years after removing the plates and screws; everything is going very well!" Other patients or future patients commented his articles. One of the comments is " $\mathrm{Hi}$, I came across your blog while searching for jaw operation reviews. I have always been fixated by a point on my face I did not know what it was until recently when I discovered that it was actually related to what seems to be retrognathia." His readers share their feelings: "In short, this evening, as I consult your blog, your articles speak to me, scare me, interest me...it is very strange!" Another example is from Pierre's blog (www.osto-bimaxpierre.overblog.com) who does not mince his words when describing how "he is slowly emerging from his black hole with pains 2-10 minutes long, strong nausea and fainting spells. At about 7:00 pm, he vomited half a liter of blood (fig. 9).

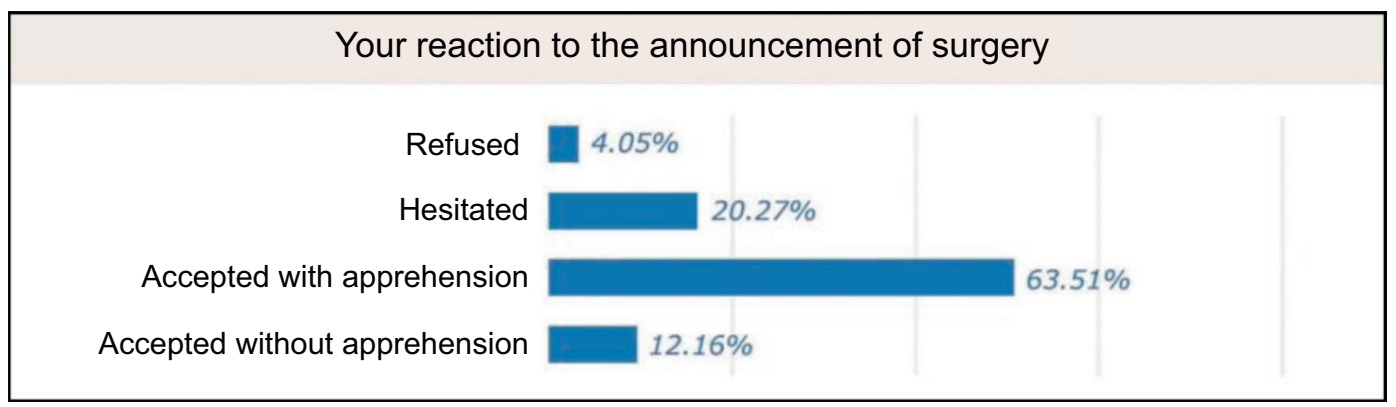

Figure 8

Statistical analysis of the fourth question on the satisfaction questionnaire. 


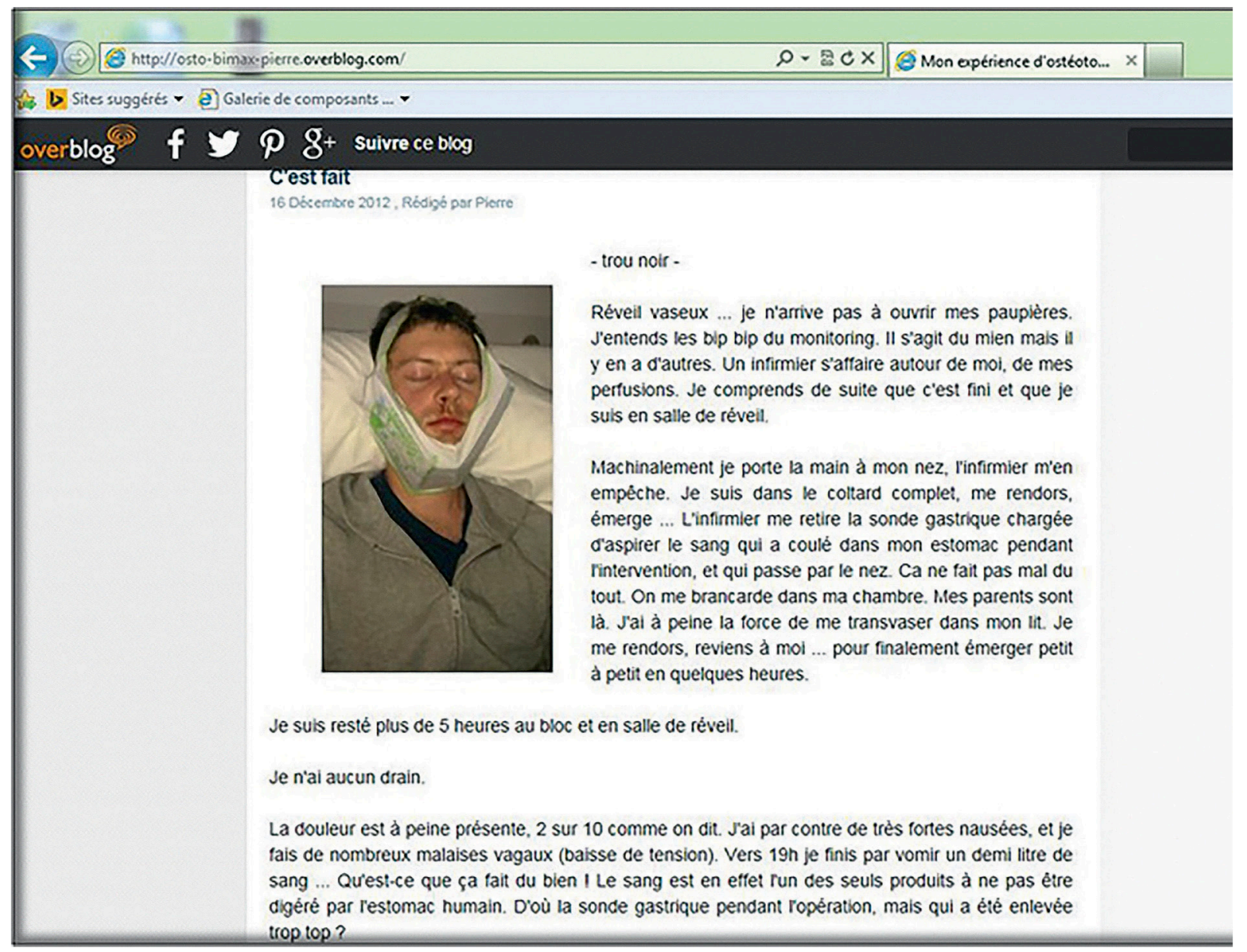

Figure 9

Extract from Pierre's blog: www.osto-bimax-pierre.overblog.com.

\section{APPROACHING PATIENTS DURING THE ORTHODONTIC SURGICAL PROTOCOL}

Once all protocols have been chosen and accepted by a patient under favorable conditions, the orthodontic preparation phase begins. This part of the protocol generally progresses smoothly; the patient is motivated and confident and regularly attends appointments; the only fear is the aggravation of the dental discrepancy.
The orthodontist continues to inform and ensure that the patient's relatives together with the other people involved in the protocol are sensitive to the oro-functional and psychoaffective aspects.

In the preoperative phase, the patient's surgery-related fears return: How long will my edema last? What 
will I be able to eat? What will be the new position of my jaw or my tongue? How long will it take to recover? We need to reinforce the information and make sure that the patient has assimilated everything by redistributing personalized and updated data rather than allowing the patient to resort to information on the Internet. The surgeon repeats the preoperative instructions before the surgery in accordance with his/her own protocol or by referring the patient to the documentation made available online by the Society of Stomatology and Maxillofacial Surgery. A downloadable brochure found on the website http://www.sfscmfco.fr/wp-content/ uploads/osteotomies.pdf recaps the postoperative effects, thus responding to the major fears that patients have, particularly concerning the intensity of pain and the edema that is often very pronounced. Indications concerning eating and intermaxillary blocking are also specified. Lastly, the society recommends that patients stop professional or scholarly activity for a minimum of 2 weeks. Consequently, for $93 \%$ of our patients, this information was satisfactory (fig. 10).

The postoperative phase is the most critical phase of the procedure, particularly the first day, according to $45.45 \%$ of our patients (fig. 11). The patient must maintain a cold, liquid diet for the first $48 \mathrm{~h}$, puréed foods for 10 to 15 days, and then, soft

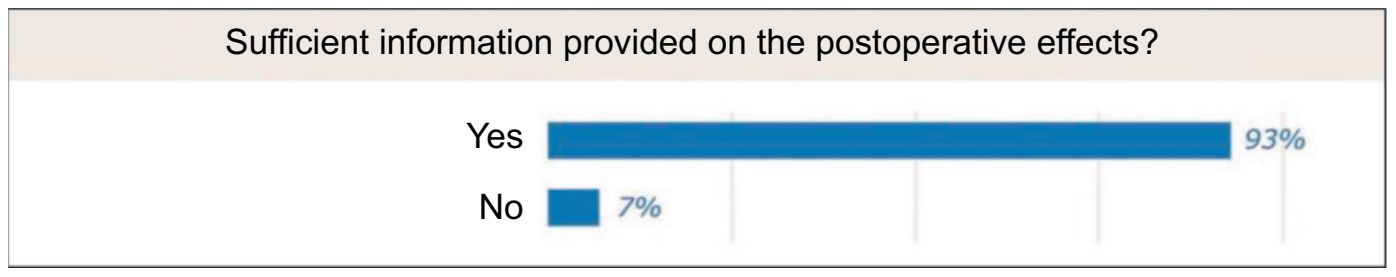

Figure 10

Statistical analysis of the fifth question on the satisfaction questionnaire.

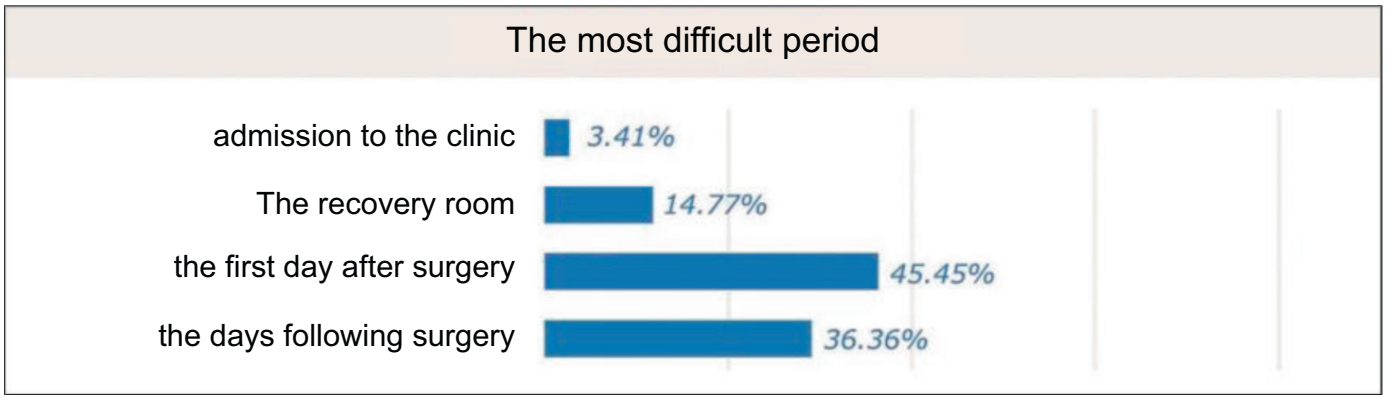

Figure 11

Statistical analysis of the sixth question on the satisfaction questionnaire. 
foods for 1 month. Most patients are therefore weakened and experience weight loss. Labiomental hypoesthesia manifests as tingling and numbness. Patients recover some of their sensitivity at a rapid rate for the first 6 weeks and then more slowly over 6 months. All these parameters justify the need for psychological support, which may be provided by relatives and/or a psychotherapist.

Reactive depression is almost systematic during the first 2 days and is spontaneously resolved on returning home. The postoperative phase is challenging because body transformation occurs suddenly. A feeling of "regression" is established due to the method of feeding and the speech difficulties encountered.

Physiotherapists or speech therapists are also essential in postoperative care. They will help reduce pain and swelling, treat lymphatic edema with massages, and will "relax" the

\section{CONCLUSIONS}

Approaching the orthodontic surgical protocol involves taking time to be better acquainted with patients and to allow them to make decisions to initiate the protocol.

This is facilitated by the proper analysis of the patient's initial request and by providing reliable sources of information. Finally, patient care must be multidisciplinary to ensure the success of the protocol both technically and emotionally.

We are also working on setting up discussion groups to be better patient's smile. They will help the patient regain sensitivity and mobility so that they can learn to speak and eat normally ${ }^{2,6}$.

During the orthodontic finishing procedure, the patient has not yet determined whether the result is satisfactory. Patients questions about the benefits of surgery. Has it helped improve confidence? Has the initial complex disappeared? In total, $90 \%$ of the surveyed patients were satisfied 6 months after surgery. The face has normally regained its original shape, and care is complete. According to studies, $24 \%$ of the patients questioned at 6 months postoperatively felt that their personality changed; $68 \%$ felt happier and 93\% were no longer fixated on their dysmorphosis ${ }^{15}$. Only $10 \%$ were dissatisfied; their disappointment was due to their excessive expectations. This dissatisfaction is often directly correlated with the patient's state of mind and psychological profile $^{1,7,8,12,13,14}$.

able to listen to patients who have already undergone surgery and to answer questions of future patients and to put them in touch with other major specialists in the orthodontic surgical protocol, namely the physiotherapist, speech therapist, psychotherapist, and even the nutritionist for postoperative dietary advice.

Conflicts of Interest: The authors declare that they have no conflicts of interest. 


\section{BIBLIOGRAPHY}

1. Arnett GW, Worley $\mathrm{C}$, The treatment motivation survey: defining patient motivation for treatment. Am J Orthod Dentofacial Orthop 1999;115:233-238.

2. Binhas E. Comment informer et accompagner efficacement un patient pour une chirurgie orthognathique ? Orthod Fr 2015;86:31-38.

3. Cunningham SJ, Harrison SD, Feinman C, Hopper C. Body dysmorphic disorder involving the facial region: a report of 6 cases. J Oral Maxillofac Surg 2000;58:1180-1183.

4. Cunningham SJ, Gilthorpe MS, Hunt NP. Ares pre-treatment psychological characteristics influenced by pre-surgical orthodontics? Eur J Orthod 2001;23:751758.

5. Finlay PM, Atkinson JM, Moos KF. Orthognathic surgery: patient expectations, psychological profile and satisfaction with outcome. Br J Oral Maxillofac Surg 1995;33: 9-14.

6. Girard M. Leroux C. Gestion des muscles et des fonctions par le kinésithérapeute dans les traitements ortho-chirurgicaux. Rééducation oro-myofonctionnelle. Orthod Fr 2015;86:95-111.

7. Hepburn S, Cunningham S. Body dysmorphic disorder in adult orthodontic patients. Am J Orthod Dentofacial Orthop 2006;130:569-574.

8. Lee S, McGrath C, Samman N. Impact of orthognathic surgery on quality of life. J Oral Maxillofac Surg 2008;66:1194-1199.

9. Oland J, Jensen J, Melsen B, Elklit A. Are personality patterns and clinical syndromes associated with patients' motives and perceived outcome of orthognathic surgery? J Oral Maxillofac Surg 2010;68:3007-3014.

10. Phillips KA, McElroy SL, Hudson JI, Pope HG Jr. Body dysmorphic disorder: an obsessive-compulsive spectrum disorder, a form of affective spectrum disorder, or both? J Clin Psychiatry 1995;56(suppl 4):41-51

11. Polo M. Body dysmorphic disorder: A screening guide for orthodontists. Am J Orthod Dentofacial Orthop 2011;139:170-173.

12. Sarwer DB. Awareness and identification of body dysmorphic disorder by aesthetic surgeons: results of a survey of American Society for Aesthetic Plastic Surgery members. Aesthet Surg J 2002;22:531-535.

13. Shaw WC, Richmond S, Kenealy PM, Kingdon A, Worthington H. A 20-year cohort study of health gain from orthodontic treatment: psychological outcome. Am J Orthod Dentofacial Orthop 2007;132:146-157.

14. Tirtley G, Decharriere-Hamzawi H, Attal J.P. Apprendre à diagnostiquer la " peur d'une dysmorphose ». Inform Dent 2005;40. Cat I.

15. Vaucard E. Impact psychologique des chirurgies orthognatiques, Mémoire pour le Diplôme d'études spécialisées en Orthopédie-Dento-Faciale, Paris : Université Paris Diderot, 2015.

16. Williams AC, Shah H, Sandy JR, Travess HC. Patients' motivations for treatment and their experiences of orthodontic preparation for surgery. J Orthod 2005;32:191-202. 